\title{
Intelligent Routing Therapist Nano Robots in a Dynamic Environment of the Human Body with the Help of Fuzzy Logic
}

\author{
Hamid Reza Naderloo', Ali Reza Naderloo*2
}

1- Department of Mechatronic Engineering, Islamic Azad University, Madjlesi Branch, Isfahan, Iran.

2- Department of Software Engineering, Islamic Azad University Yazd Branch, Yazd, Iran.

\section{ABSTRACT}

Introduction: Using nanotechnology, scientists have been able Nano robot knowledge that the human body is designed to be therapeutic and protective role and play therapist. This Nano robot could help in different ways such as a capsule or injection into the vascular system of the body, the human body and controlled or programmed manner (smart) to play the role of a therapist. . These robots can perform a routing optimization in the shortest time and shortest distance traveled, upon detection of possible damage, damage to the site and using nanotechnology to repair it.

Material and Methods: In this class of problems to find the path or paths (preferably optimum), which move the robot without colliding with obstacles static/dynamic of a specific origin to its destination. In this paper presents a simulation system under manual control, wireless routing protocol, and is used for navigation Nano robot because of the problems raised in writing and to fix bugs of the system, an intelligent routing system, To move Nano robot therapist, based on the fuzzy control system in which the steering motor in the vicinity of obstacles and destination point for platform Nano robot be issued.

Results: For the more accurate and more complete routing in dynamic environments without the structure of the human body (the restrictions are not predetermined and fixed), need to work on issues relating to robot motion planning and to determine the optimal path will be moved. This research finally revealed that a system following the fuzzy logic for a mobile Nano robot navigation.

Conclusion: The present findings from the implemented designs here took into account the merits and demerits and evaluated the optimal navigation issue in robots following the fuzzy logic and the genetic algorithm. We found out that to solve such issues more efficiently.

(i) Please cite this article as:

Naderloo HR, Naderloo AR. Intelligent Routing Therapist Nano-Robots in a Dynamic Environment of the Human Body with the Help of Fuzzy Logic. Iran J Med Inform. 2016; 5(1): 12-18. DOI: 10.24200/ijmi.v5i0.148

\footnotetext{
* Corresponding Author: AR Naderloo, Department of Software Engineering, Islamic Azad University, Yazd Branch, Yazd, Iran (Email: alir_naderlo@yahoo.com)

\author{
Article History \\ Received: 2015-11-22 \\ Accepted: 2016-01-31 \\ Published: 2016-03-15 \\ Keywords \\ Fuzzy Logic \\ Nano-Robot \\ Intelligent Routing
}




\section{INTRODUCTION}

Imagine a doctor who wishes to treat a patient. Rather than prescribing a tablet or shot, he sends the patient to a center which would inject a tiny robot into the patient's veins. The robot would reveal the cause, move within the body and pave the way for fixing or removing the cause directly [ 1$]$.

Nano robots need to be tiny and active so that they can move easily in the complex network of veins and arteries without meeting any obstacles (mobile or immobile). A Nano robot should be able to carry miniature devices. Assumed not to remain in body for long, robots are expected to exit body in a way. It is also known that Nano robots are capable of treating certain diseases under uncertain circumstances [1].

Doctors and engineers believe that when these robots are so small in size that can carry therapeutic devices on them, thy can be used much more effectively than the traditional method. As an instance, a doctor may prescribe antibiotics so that the patient's immune system is strengthened. Once dissolved in water, antibiotics begin their journey in body veins and reach infectious spots. However, Nano robots can directly move to the infectious spot and inject the drug. Then, the patient would feel less pain than the side effects of a drug [1].

Overall, making sure of the proper control of such Nano machines has been open to controversy in the current world of Nano robots. Nano mechatronic methods can be employed to evaluate and estimate this effect.

One key issue in the design of such mobile robots is their proper control, direction and navigation. A robot needs to be directed toward a particular target and refrain from any obstacle on the way. It is expected to take the shortest path to the target (optimal path) for movement. Space control is another key dynamic aspect of a robot. Thus, to control space, a key issue is the unknown dynamic of the environment $[\underline{2}, \underline{3}]$.

Generally speaking, despite many recent advancements in robot navigation in different settings, yet there are many issues left unsolved. These are mainly caused by the nature of real environments and a series of unknown factors that exist in such settings. In such applications, first of all, there is little or ambiguous knowledge of how a robot works. Secondly, the sort of information collected from the environment is often unreliable as the limited range of environmental sensors, some environmental effects (e.g. appearance of an obstacle in the way of the sensor) and observation issues (e.g. less light) often lead to imprecise information. These errors might intervene in the data interpretation process and lead to false decisions. Thirdly, actual environments are often marked by complicated and unpredictable dynamic features $[\underline{2}, \underline{4}]$.

To act in actual settings, robots should be capable of making safe movements and pursue the preset goals successfully despite the probable sudden changes (in dynamic settings). Real environments are hardly ever totally known to us and predictable. Therefore, defining the precise directions in advance to the movement does not often work in such public contexts. Defining the directions of a robot can be analyzed in two subcategories $[\underline{5}, \underline{6}]$.

Pursuing the goal: is a general concept as short distances cannot be traced using only the local information. Therefore, the topology of space is the main issue in finding the best way to the goal. Avoiding obstacles: is often attainable using only the local information. In other words, a robot should first sense the obstacles so as to avoid them.

Finally, the solutions to the planning issue of robot navigation and movement can be categorized in two methodologies: classic and exploratory [7]. The former would deal with such problems as computational complexities at higher levels of space configuration. Moreover, certain solutions such as artificial potential fields are local and can trap robot in local minimal points (slightly bigger than the primary minimal points and located near the movement path). The latter (including the fuzzy control system) has been suggested to improve their performance.

\section{MATERIAL AND METHODS}

\section{Nano robot therapists}

Using Nano robots does not mean to replace doctors' role during a therapy. To the contrary, a robotic procedure needs to be done under the supervision of medics. Thus doctors play the role of a pilot all along the path [].

Soon Nano robot therapists enter different medical fields such as surgery. Equipped with surgical devices and prepared for advanced movements, these robots are capable of operating on the inner and outer surface of cells, often inaccessible to humans. These Nano robots are well adapted to biological systems and are capable of locating cancerous cells and destroying them. They will be designed in a way to be capable of removing venal blockage and repairing damaged cells. Moreover, these micro-surgeons can make transplants to tissues.

How Nano robot surgeons work is being investigated. Their advent to the realm of science will for sure make great revolutions. A sample of nanorobot therapists (surgeons) is indicated in Fig 1.
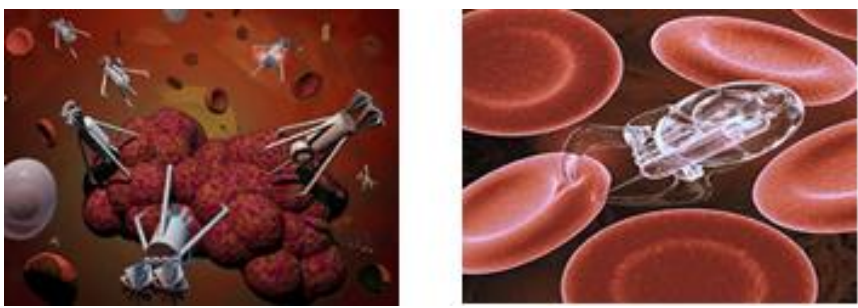

Fig 1: Nano robot therapists [] $]$

Overall, huge robots of hanging arms are not the only hallmarks of the medical world in future. Rather, robots of the smallest size can be effectively employed in surgeries. Mini-robots than can enter body might be even expected to conduct sensitive surgeries and then exit body without any need to make cuts in organs and open up the body.

These systems possess certain merits and demerits. The latter might include the longevity of Nano robot (theoretically speaking, lasting for years), short duration of performance (due to their limited movement and the concomitant biological events happening in shorter time) and limited size (sets certain limits as the very small size of the robot makes it capable of passing any definite or indefinite pathway nonstop). It is even possible that a Nano robot, due to its miniature size, is recognized as a disease factor by body.

It is noteworthy that to work best within human body, the size of a Nano robot therapist should not exceed 3 microns in diameter [8]. These Nano robots should contain efficient and cost-effective transducers and actuators so as to interact with the environment as soon as they enter the body.

Fig 2 indicates how Nano robots are used in heart surgeries to open up blocked or damaged vessels and to take samples or inject stem cells into hart cells. Fig 3 represents several types of Nano robot therapists. 


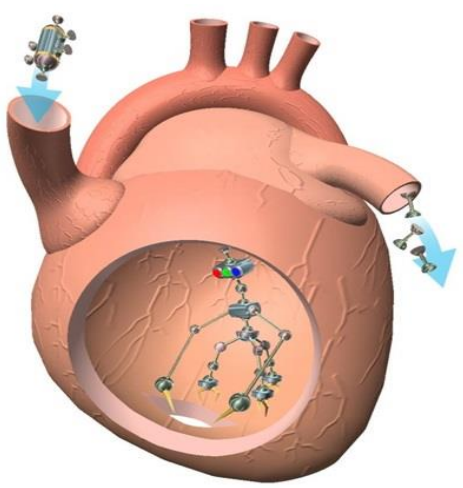

Fig 2: Nano robots in heart surgeries

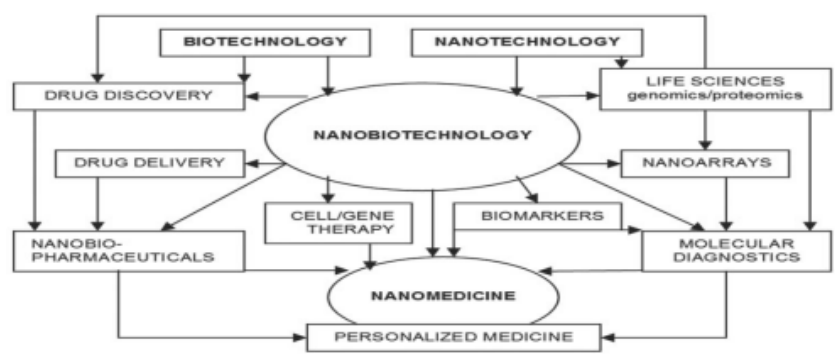

Fig 3: Nano robot application

Fuzzy logic

The theory of fuzzy sets and fuzzy logic was firstly put forth by professor Lotfizadeh in a dissertation entitled as "The fuzzy sets: information and control" in 1965. Generally speaking, the components of a fuzzy logic argument can be conceptualized. As the system input is an absolute numerical value (Crisp), the task of the fuzzification is to turn the input into a fuzzy set. The membership function information is included in the Data Base. The Rule Base contains rules and regulations that would determine the output of a fuzzy system [9].

In fact, the fuzzy logic is based on the theory of fuzzy sets which is a generalization of the classic theory of sets in mathematics. In the classic theory of sets, any element either belongs to the set or not. In fact, the membership of the elements would follow a binary 0 or 1 pattern. However, the theory of fuzzy sets generalizes this concept and put forth the graded membership. Accordingly, each element can belong to a set to a certain degree and not necessarily completely. In this theory, the membership of the elements in any set is determined according to the function $\mu(\mathrm{x})$ in which $\mathrm{x}$ represents a certain member and $\mu$ stands for a fuzzy function which would determine the membership degree of the member $\mathrm{x}$ in the target set. It varies between 0 and 1 . In other words, $\mu(x)$ would generate a mapping of $x$ values on 0 -to-1 numerical values. The $\mu(\mathrm{x})$ function may be a collection of discontinuous or continuous values. The former is when $\mu$ is comprised of only some discontinuous values between 0 and 1 for example it can include .3, .5, .7, .9, 0 and 1 . However, when the set of $\mu$ values is continuous, a continuous curve is formed of decimals between 0 and 1. Fig 4 displays diagram of continuous values mapped on $\mu(\mathrm{x})$. The $\mu(\mathrm{x})$ function in this figure can define the membership rule in a hypothetical fuzzy set []].

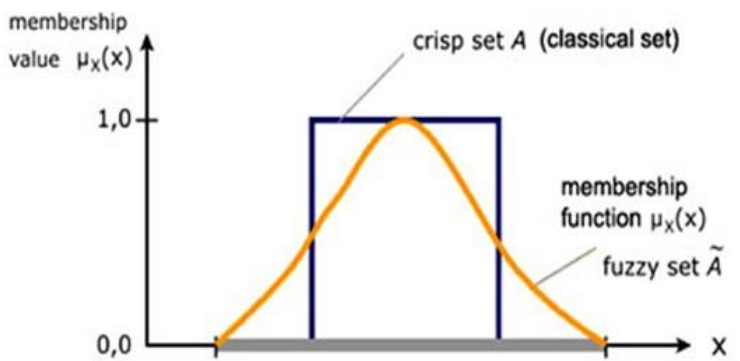

Fig 4: Diagram of continuous values mapped [9]

The fuzzy logic can be employed through certain rules known as 'fuzzy operators'. These rules are often defined as IF variable IS set THEN action. As an instance, assume that we wish to provide a fuzzy $\mu(\mathrm{x})$ function. Fig 5 shows how the temperature is mapped on several fuzzy sets: cold, cool, moderate, warm and hot. As it can be observed, any particular temperature can belong to one or two sets [7].

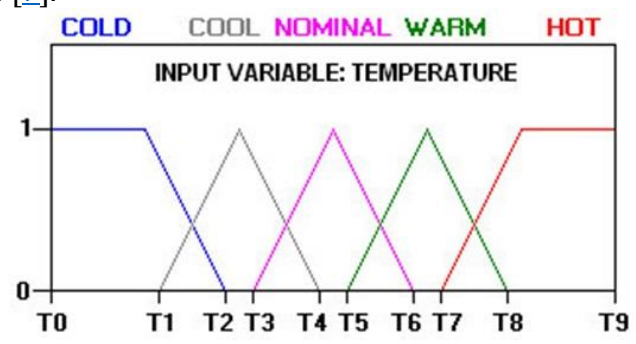

Fig 5: The temperature is mapped on several fuzzy sets [9] As an instance, temperatures ranging from $\mathrm{T} 1$ to $\mathrm{T} 2$ belong both to the cold and cool set. However the degree of membership to either is different. The temperature close to T2 only belongs to the cold set for a couple of decimals whereas it is much closer to the percentage node in the cool set.

In 1975, two fuzzy logic scientists, Mamdani and Assilian developed the first actual fuzzy control [7]. They solved this problem by estimating the center of gravity (C) of the area of the mixed two trapezoids that can be viewed in Fig 6. They mapped it on axis $t$ and estimated Tx2. Like the classic logic, the fuzzy logic has got several basic operators. Such operators as AND, OR and NOT are used in the classic logic.

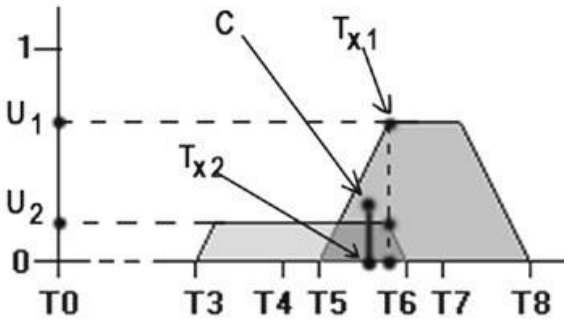

Fig 6: mapped on axis $t$ and estimated $T \mathrm{x}_{2}$

So far, we only dealt with the fuzzification process. Once the fuzzy operation is implemented on the system, we should eventually reach a crisp. To do the defuzzification, often either the center of gravity (COG) or the center average (CA) method is used.

Simulation of nanorobot therapists' navigation as a manually-controlled system via a wireless communication channel

Overall, in this navigation, the doctor is located at a certain station (the master device) from which s/he controls the navigation of the robot simply and manually [2]. The doctor is seated on a unit and watches the videos and controls the process via X-ray and a magnetic navigator not unlike playing a computer game using a joy-stick. Here, a mouse is used by the doctor to control the 
movement of the Nano robot therapist along the path. In fact, the hand movements is simulated to match the navigation of the Nano robot therapist.

The doctor's hand movements is conveyed through a transmitter channel (wireless communication) to the slave to control the pathway []․ The communication channel (transmitter) is located between the doctor's console (the master) and the Nano robot therapist (the slave).

To examine and simulate how a doctor manually controls a Nano robot therapist inside the dynamic environment of body from a distance, MatLab was used to create a 2D simulation of a Nano robot control and navigation through a wireless communication channel (for instance on two distinct computer systems one as a master and the other as the slave connected). In this sample, we can observe how a Nano robot therapist moves in a 2D space when the source and destination of movement is set by a hand movement of the controlling doctor. A left-click on the mouse (the controller) would suffice to change the direction to left and a right-click to change it to the right, all transmitted through a wireless navigation port. Fig 7 indicates the components of the manually controlled 2D pathway along which the Nano robot moves.

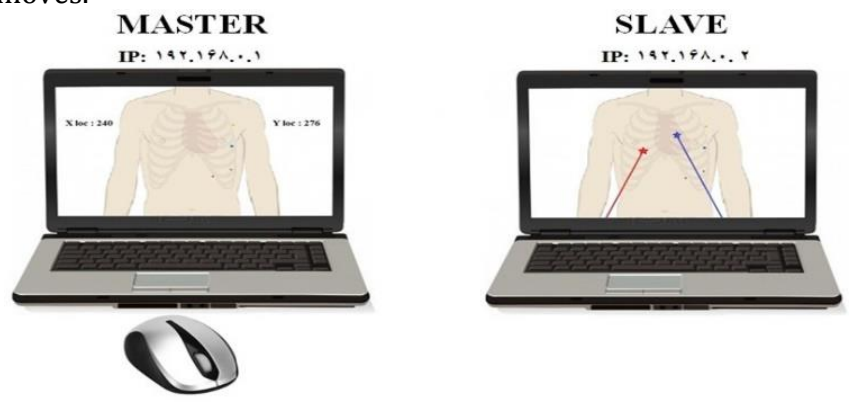

Fig 7: The components of the manually controlled 2D pathway The procedure goes as the $2 \mathrm{D}$ coordinates $(\mathrm{X}, \mathrm{Y})$ are sent through the wireless port of the network to the slave which results in a red-colored line. This would show the source of the robot movement. For the movement along the path to reach the destination, a similar procedure is followed. The graphical space has been designed by GUIDE in MatLab which helps to follow the movement of the doctor's hand movement and records the right or left clicks sent through the network wireless port. This plan was piloted on a local wireless network. The Ping command revealed that there was no missing data in that local network and the rate of data transmission within the network was found to be 100 Mpbs. However, later on when in practice both the master and slave devices were simultaneously monitored in the network, the procedure was done with a significant delay (partly due to MatLab). Even to test the transmission of images, a DVR device as well as an IP camera were installed within the local internal network. No particular image processing was done on the images. Similarly, the transfer of images was seen to be delayed within the network between the master and slave devices and the images were thus observed slowly and with delay.

Therefore, there were several probable problems facing this plan including the wireless communication limitations (e.g. band breadth, delay, etc.), limited view to control and guide the Nano robot inside body and working at the Nano scale. These all would threaten a patient's health. Thus, there should be other ways to consider such as smart navigation.

Smart navigation of Nano robot therapists in dynamic human body using the fuzzy logic:

For the sake of navigating unknown environments and facing mobile or immobile obstacles (inside human body), without a knowledge of the exact position and movement function of the obstacles, the movement must be made node to node. Thus, momentary navigation methods should be followed. The fuzzy logic is highly efficient in the momentary node to node navigation [10].

In the classical methods, considering the certainty of the input, modelling is done via pre-defined functions and the relevant parameters are estimated through interpolation algorithms [2]. However, in this part of research, we are faced with the uncertainty of input investigated through the fuzzy logic which is a formal methodology for implementing perception-based tasks [2].

To do so using a fuzzy system, the navigation is done from the source in a node to node manner until the robot reaches its target destination. In this approach, every moment, the next node is selected among 8 options (in this research, the hypothetical pathway is considered as 8). To find the pathway more precisely, it can be considered as 16 or even more. The rest followed the fuzzy logic as indicated in Fig 8.

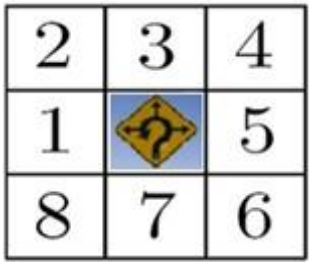

Fig 8: The pathway for new nodes

The fuzzy input taken into account in navigation are:

- $\quad$ the angle difference of the next nodes and the target

- $\quad$ the distance of the next nodes from the nearest obstacle It is noteworthy that every moment, in any node that the robot is located, we can know about the position of obstacles only to a certain radius of the robot sensors [2]. As an instance, if the sensors can only sense through 30 centimeters around (in the simulation by MatLab, the default radius is taken as $30 \mathrm{~cm}$ ). In other words, every second, the robot would be aware of what happens around only as far as 30 centimeters. At each moment of navigation, the next node would be selected among the next 8 nodes each with an estimated priority coefficient as the wouldbe-chosen node (through the fuzzy logic).

Once the fuzzification is done, each input node would be followed by an output representative of the priority of selecting that same node. In fact, the next node would be the one of the highest priority coefficient. The overall phases of momentary and node to node navigation with the help of the fuzzy logic.

Fuzzification of the inputs

Navigation via the fuzzy logic is done node to node and momentarily. In fact, the fuzzy logic is done as frequently as the number of nodes along the way [10]. Two fuzzy inputs have been used for the navigation purpose in this system: angle difference with the target destination and the distance from the nearest obstacle. For each input, 5 membership functions have been used for fuzzification. The membership functions have been taken here as hypothetical). In Fig 9 and 10, we can see the membership functions for the angle input and the distance input. 


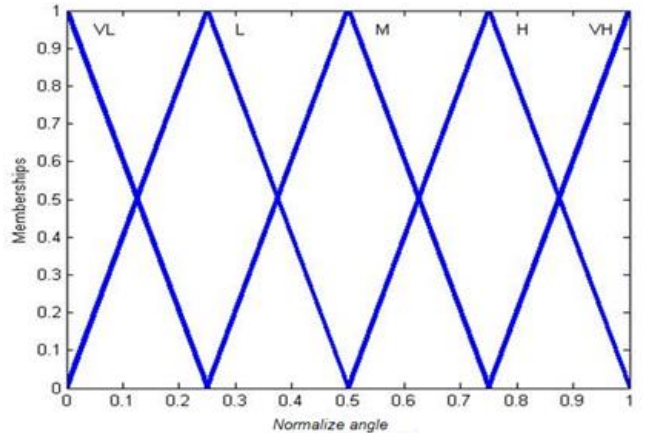

Fig 9: The membership functions for the angle input [10]

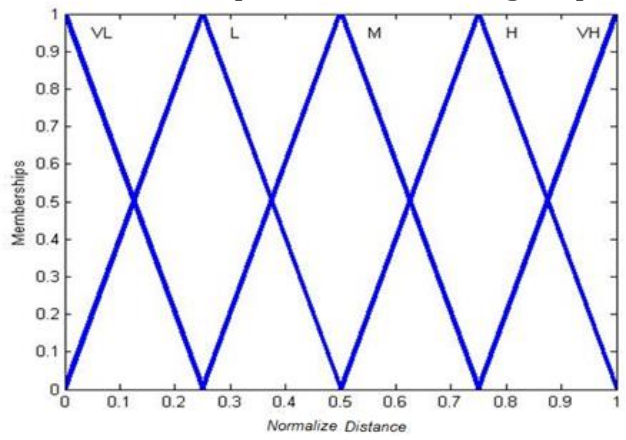

Fig 10: The membership functions for the distance input [10] It is noteworthy that momentarily the non-fuzzy input for each next 8 nodes get normalized and reported as $0-1$. Only then, the fuzzification follows. In this sytem, for the put-put, 5 membership functions are used as the following [10]:

- $\quad$ Very Low (VL)

- $\quad$ Low (L)

- $\quad$ Moderate (M)

- $\operatorname{High}(\mathrm{H})$

- $\quad$ very High $(\mathrm{VH})$

The overall procedure begins with the estimation of the shortest distance of every node to the nearest obstacle as well as the angle difference to the target destination normalized between 0 and 1 . As the next step, for each and every node, the normalized nonfuzzy input gets fuzzified from the nearest existing obstacles as well as the angle difference from the target destination using the relevant fuzzy membership functions. The membership functions for the fuzzy output is as in Fig 11.

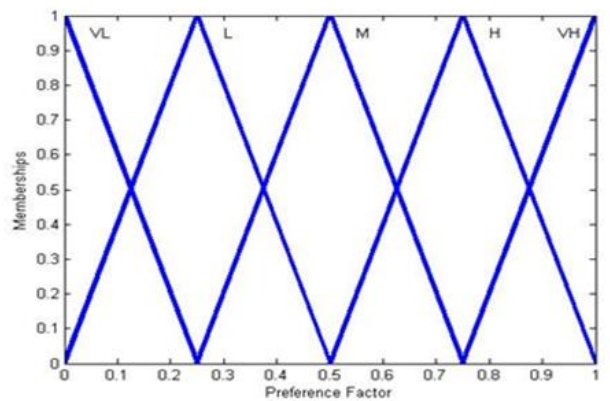

Fig 11: The membership functions for the fuzzy output [10] Table of the fuzzy rules

As mentioned earlier, each next node is selected in the pathway through the fuzzy logic. The fuzzy rules for selecting the next node in momentary navigation are indicated in Table 1 . According to this table, the more the distance from the nearest obstacle and the lower the angle difference from that of the target, the higher the probability of that node to be selected as the next node along the pathway.
To find the fuzzy output in each node, reference can be made to the table of fuzzy rules which contains all the rules as AND (hypothetical rules). In the next step, the fuzzy output can be conceptualized as a fuzzy output through Mamdani's multiple method or Max-Min composition method [2]. In this step, one or more of the rules within Table 1 are implemented in accordance with the fuzzy input of each node.

Defuzzification and setting the final priority coefficient As mentioned earlier, the most common defuzzification methods are the Center of Gravity (COG) and the Center of Average (CA) method [9]. To design the present nanorobot navigation system, the latter method was used for defuzzification. Eventually, once the defuzzified output is determined from among the existing 8 options, the option with maximal defuzzified output is selected as the next node along the pathway. The above procedure goes on until the robot reaches the target destination. Fig 12 includes how the optimal path is decided upon and how the mobile and immobile obstacles are faced with in 6 hypothetical steps.

Table 1: The fuzzy rules for selecting the next node in momentary navigation

\begin{tabular}{|c|c|c|}
\hline $\begin{array}{l}\text { Output: the higher the } \\
\text { probability of that node }\end{array}$ & $\begin{array}{c}\text { the lower the angle } \\
\text { difference from that } \\
\text { of the target }\end{array}$ & $\begin{array}{c}\text { the distance } \\
\text { from the nearest } \\
\text { obstacle }\end{array}$ \\
\hline$(\mathrm{M})$ & $(\mathrm{VL})$ & $(\mathrm{VL})$ \\
\hline$(\mathrm{L})$ & (L) & (VL) \\
\hline$(\mathrm{VL})$ & $(\mathrm{M})$ & $(\mathrm{VL})$ \\
\hline$(\mathrm{VL})$ & $(\mathrm{H})$ & $(\mathrm{VL})$ \\
\hline (VL) & $(\mathrm{VH})$ & (VL) \\
\hline$(\mathrm{M})$ & (VL) & $(\mathrm{L})$ \\
\hline (M) & (L) & (L) \\
\hline$(\mathrm{L})$ & (M) & $(\mathrm{L})$ \\
\hline (L) & $(\mathrm{H})$ & (L) \\
\hline$(\mathrm{VL})$ & $(\mathrm{VH})$ & (L) \\
\hline$(\mathrm{H})$ & $(\mathrm{VL})$ & (M) \\
\hline (M) & (L) & (M) \\
\hline (M) & (M) & (M) \\
\hline (L) & $(\mathrm{H})$ & (M) \\
\hline$(\mathrm{VL})$ & $(\mathrm{VH})$ & $(\mathrm{M})$ \\
\hline$(\mathrm{VH})$ & $(\mathrm{VL})$ & $(\mathrm{H})$ \\
\hline$(\mathrm{VH})$ & (L) & $(\mathrm{H})$ \\
\hline$(\mathrm{H})$ & (M) & (H) \\
\hline$(\mathrm{M})$ & $(\mathrm{H})$ & $(\mathrm{H})$ \\
\hline$(\mathrm{L})$ & $(\mathrm{VH})$ & $(\mathrm{H})$ \\
\hline$(\mathrm{VH})$ & $(\mathrm{VL})$ & $(\mathrm{VH})$ \\
\hline$(\mathrm{VH})$ & $(\mathrm{L})$ & $(\mathrm{VH})$ \\
\hline$(\mathrm{H})$ & (M) & $(\mathrm{VH})$ \\
\hline (M) & $(\mathrm{H})$ & $(\mathrm{VH})$ \\
\hline (M) & $(\mathrm{VH})$ & $(\mathrm{VH})$ \\
\hline
\end{tabular}




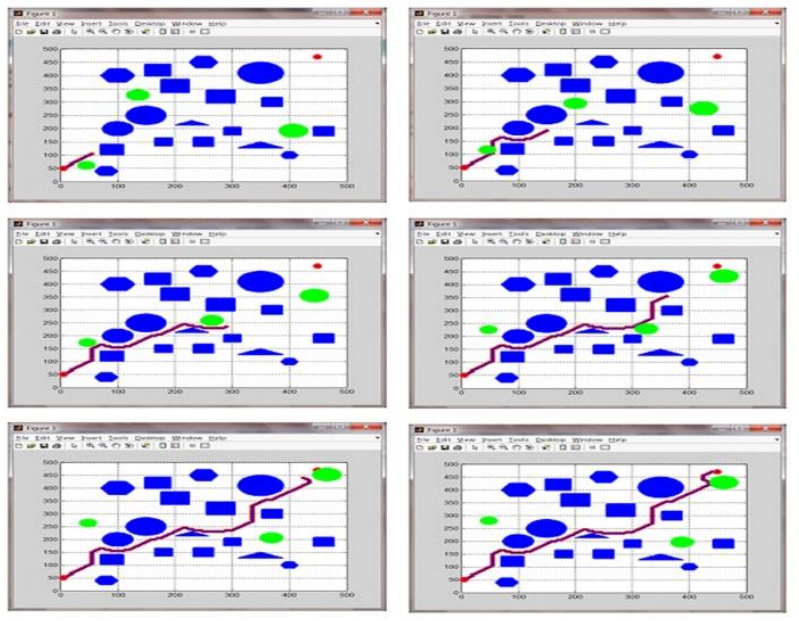

Fig 12: Optimal path decision

Table 2 includes the results of the length and time duration of the final path selected through the fuzzy logic in different contexts using the 8 sample enlisted.

\section{RESULTS}

The fuzzy logic is not inherently parallel and can only search the space of a given problem along one direction at any given moment (in real time). if the final solution is a local optimal one or a subcategory of the main solution, anything done so far needs to be discarded and the procedure should start anew [10]. This research finally revealed that a system following the fuzzy logic for a mobile Nano robot navigation:

required no prior knowledge of the environment (useful to be employed in unknown environments)

- momentarily was able to recognize its periphery (in a radius of $30 \mathrm{~cm}$ ) which was time-effective and useful for optimization. In fact, it was capable of recognizing the spatial position of (mobile or immobile) obstacles within 30 $\mathrm{cm}$

was aware of the target coordinates

- followed a simple and comprehensible methodology in problem-solving

would successfully combine input parameters (the angle difference of the next nodes and the target and the distance of the next nodes from the nearest obstacles)

conveniently control and set the output parameters using the fuzzy rules

Table 2: The results of the length and time duration of the final path.

\begin{tabular}{|c|c|c|c|}
\hline \multirow[b]{2}{*}{ Start } & \multirow[b]{2}{*}{ Goal } & \multicolumn{2}{|c|}{ Fuzzy Logic } \\
\hline & & $\begin{array}{c}\text { Time } \\
\text { duration (s) }\end{array}$ & $\begin{array}{c}\text { length } \\
\text { duration }(\mathrm{cm})\end{array}$ \\
\hline$(50 \cdot 5)$ & $(470 ، 450)$ & 68.5900 & 738.7610 \\
\hline$(20 \cdot 55)$ & $(460 ، 455)$ & 70.8574 & 760.832 \\
\hline (30،65) & $(465 ، 460)$ & 75.3923 & 813.2590 \\
\hline (50،100) & (470،470) & 68.0231 & 704.7666 \\
\hline (25، 150) & (450، 400 ( & 57.8196 & 579.5584 \\
\hline (15،110) & (450، 450$)$ & 72.5580 & 785.3301 \\
\hline$(5 ، 60)$ & (480، 480) & 70.2905 & 768.2590 \\
\hline (20،150) & (460، 490) & 91.8312 & 981.0408 \\
\hline
\end{tabular}

\section{DISCUSSION}

To conduct such navigations in unknown environments at the presence of mobile or immobile obstacles, without any knowledge of the coordinates and movement function of the obstacles, we can use the genetic algorithm. Due to the fact that the length of the pathway is variable, we can also use the variable chromosome structure (since the different pathways are of different length) [11]. In other words, each gene would determine the number of a mid-node along the way (i.e. the path selected by each chromosome). That is to say that always the first gene indicates the start point and the last gene points to the target destination.

Firstly, an initial randomized population of chromosomes is generated. Once the outcome solutions are analyzed in each iteration, a number of the best chromosomes are selected as the parents for the next generation. Then, the integration and mutation process is done on the parents to further generate the next generation of chromosomes. This process continues up until the time that the termination conditions are met. The navigation steps are taken until the output is obtained.

The first advantage of the genetic algorithm is access to the overall optimal point instead of the local optimal point [12]. That is to say that always to a great extent we can trust the outcome of this algorithm and that the solution it offers can be probable the best. The genetic algorithm can search the problem space from multiple aspects in only one moment. If one way does not prove effective, others will be tried and more resources will be available. However, the main disadvantage of this algorithm is that despite its easy implementation it is highly costly [11].

Often, problem-solving requires the production of a multiple thousand generations of chromosomes and this problem demands much time (especially if the size of the initial population is high and the function goal is complicated). It occasionally takes more than a week for a Pentium processor to solve a certain problem. This is for sure more than long for problem-solving and this would make the use of algorithm problematic sometimes. Therefore, the present algorithm provides the optimal solution in a longer time and using many more sources as in using the genetic algorithm, the robot pathway is designed in a developmental process (coming to recognize the pathway) and only then the start-to-move command is given. This would require more sources to be used [12]. As a consequence, along the pathway, the fuzzy algorithm would take less time while the genetic algorithm takes a shorter distance.

\section{CONCLUSION}

The present findings from the implemented designs here took into account the merits and demerits and evaluated the optimal navigation issue in robots following the fuzzy logic and the genetic algorithm. We found out that to solve such issues more efficiently, we suggest using an integrated system of genetic and fuzzy algorithms (optimizing the parameters of a fuzzy system via the genetic algorithm). In fact it is suggested in this approach that the navigation be done momentarily using the fuzzy logic. In other words, optimizing the sample pathway takes the least time possible. Meanwhile, we can only once and non-momentarily use the genetic algorithm offline to set the different parameters in a fuzzy system. As an instance, genetic algorithm can be used for the optimal setting of the membership functions used in the fuzzy logic as well as the optimal setting of the fuzzy logic table of rules. This would provide for all the advantages of the fuzzy logic 
simultaneously in optimizing the Nano robot therapists' navigation.

In other words, if a combination of the genetic and fuzzy systems is used we can optimally benefit from the two main factors involved in moving along the pathway in the least time possible and through the shortest distance. Finally, an ideal and optimal path will be found in terms of the main factors of time and distance for the target navigation.

\section{REFERENCES}

1. Nasiri M. New technologies. Med \& Lab Engineering Magazine. 2011; 132(11): 8-11.

2. Alavi SM, Ghanbarzadeh A, Alavi SE, Ghanavati M. Using fuzzy method to robot routing based on sensors' values. Proceeding of $1^{\text {st }}$ National Conference of Machanical Systems Simulation. Ahvaz, Iran. 2011.

3. Borenstein J, Everett HR, Feng L. Navigating mobile robots: Systems and techniques. A.K.Peters Ltd.; Wellesley. 1996.

4. Barraquand J, Langlois B, Latombe JC. Numerical potential techniques for robot path planning. IEEE Transactions on Systems, Man, and Cybernetics. 1992; 22(2): 224-41.

5. Maaref $H$, Barret C. Sensor-based navigation of a mobile robot in an indoor environment. Robotics and Automation Systems. 2002; 38(1): 1-18.

6. Storn R, Price K. Differential evolution: A simple and efficient adaptive scheme for global optimization over continuous spaces. Journal of Global Optimization. 1997; 11(4): 341-59.

7. MotieGhader H, Lotfi Sh, Seyed Asfehlan MM. Intelligent optimization methods. Azad Islamic University Publication; Shabestar. 2010.

8. Cavalcanti A, Freitas RA Jr. Nanorobotics control design: A collective behavior approach for medicine. IEEE Trans on NanoBioScience. 2005; 4(2): 133-40. PMID: 16117021 [PubMed]

9. Yang X, Moallem M, Patel RV. An improved fuzzy logic based navigation system for mobile robots. Proceedings of Intelligent Robots and Systems Conference. Las Vegas, USA. 2003.

10. Pholsiri C, Kapoor C, Tesar D. Real-time robot capability analysis. Proceeding of Computers and Information in Engineering Conference. Long Beach, Canada. 2005.

11. Petrenko YN, Alavi SE. Fuzzy logic and genetic algorithm technique for non-linear system of overhead crane. IEEE Region 8 International Conference on Computational Technologies in Electrical and Electronics Engineering (SIBIRCON), 2010.

12. Goldberg DE. Genetic Algorithms in Search, Optimization and Machine Learning. Addition-Wesley Professional; 1989. 